\title{
Fault Detection in an Induction Motor Drive Using Discrete Wavelet Packet Transform
}

\author{
Geraldine Mathew ${ }^{1}$ \\ ${ }^{I}$ Department of Electrical Engineering, RGCER, Nagpur
}

\begin{abstract}
The aim of this study is to employ the wavelet technique for diagnosis of open-circuit and short circuit faults in the dc link of Induction motor drive. The symlet wavelet is selected as the wavelet base to perform stator current analysis during faults. In this method, the stator currents are used as an input to the system. The MATLAB software is used to process discreet wavelet transform (DWT) of the signals. The stator current is used for the detection of the fault. When an open-circuit fault or a short circuit fault appears, the signal fault information is included in each frequency region. The time of the spikes in the DWT is correlated with the time of fault. As a result of time domain \& frequency studies, a faulty system can be easily discriminated from a healthy one
\end{abstract}

Keywords: DC link faults, Wavelet, Symlet, and Fast Fourier transform.

\section{Introduction}

The modern day advanced engineering systems have become more complex. As a result, there has been a growing demand for reliable fault detection and identification and diagnosis schemes .A fault is defined to be any deviation of the system outside its designed operation. Fault detection is the process of recognizing when a system has begun to operate outside its original design limit. Fault identification is the process of locating the most likely root cause of the detected failure within the system's components or subsystems. A survey of system fault identification and diagnosis can be found in [1] [2] [3]. Since the field of fault diagnosis has been developed, new techniques have emerged to monitor power systems and give immediate information as a fault occurs. Fault detection and diagnosis for power system is complicated because of the wide variations in power components operating conditions and performance caused by many different constraints. The theory of application of wavelet transforms (WT) to fault detection and classification has been discussed in many papers. The induction motor is widely used in the electromechanical energy conversion devices due to their low cost, ruggedness, low maintenance, and operation with an easily available power supply. Since the induction motor current contains harmonics it can be used for the identification of many faults. Many different techniques are employed in fault diagnosis in drives. One such technique is wavelet technique. This technique is new in the field of fault diagnosis due to its ability to extract information in both time and frequency domain as well as it provides a sensitive means to the diagnosis of faults if compared to other signal processing method like Fourier Transform.

The fault diagnosis has two main levels:

1) It comprises a traditional control level

2) It contains a knowledge based fault diagnosis

One of the most important analysis tools in both frequency and time domain is the wavelet. The multi resolution analysis and good time localization makes wavelet very attractive for research work in fault diagnosis. Wavelets are localized in both the time and frequency domains because wavelets have limited time duration and frequency bandwidth.

\section{Overview Of Wavelet Transform}

Fourier analysis techniques provide significant information on frequency components of signals under study, but offer no information regarding where a particular frequency was located in the time axis. In contrast, wavelet transforms offers time-frequency information of signals under study, thereby making wavelet transform methods more comprehensive than Fourier transforms. In signal analysis, wavelet coefficients, at a first level of decomposition, are obtained from a signal under analysis by applying a mother wavelet. The process can be repeated if the mother wavelet is scaled and translated. The mother wavelet function (denoted by $\psi(t)$ ) and its scaling function (given as $\phi(t)$ ) describe a family of functions which are required to satisfy a number of criteria. It must have a zero mean denoted in equation below

$$
\int_{-\infty}^{+\infty} \psi(t) d t=0
$$


In addition $\Psi(t)$ must have a square norm of one as denoted in equation (2)

$$
\int_{-\infty}^{+\infty}|\psi(t)|^{2} d t=1
$$

The requirements are ensured by having a mother wavelet that is absolute and square integrable. The mother wavelet forms a family of wavelets when the function is scaled and translated in the time domain. When a mother wavelet is translated by a factor of ' $a$ ' and scaled by a factor of ' $b$ ', it can be expressed in a generic form as follows

$$
\phi_{a, b}(t)=\frac{1}{\sqrt{a}} \phi \frac{(t-b)}{a}
$$

The use these wavelet functions provide a robust method of analysing non stationary signals to provide both frequency and time information. Wavelet transformation contains many kinds, but in this paper discrete wavelet transform \& continuous wavelet transform has been discussed.

\section{A. Discrete wavelet transform}

The wavelet transformation is processes of determining how well a series of wavelet functions represent the signal being analysed. The goodness of fitting of the function to the signal is described by the wavelet coefficients. The result is a bank of coefficients associated with two independent variables, dilation and translation. Translation typically represents time, while scale is a way of viewing the frequency content. Larger scale corresponds to lower frequency implying better resolution. The most efficient and compact form of the wavelet analysis is accomplished by the decomposing a signal into a subset of translated and dilated parent wavelets, where these various scales and shifts in the parent wavelet are related based on powers of two. Full representation of a signal can be achieved using a vector coefficient of the same length as that of the original signal.

Considering a signal consisting of $2 \mathrm{~m}$ data points, where $\mathrm{m}$ is an integer. DWT requires $2 \mathrm{~m}$ wavelet coefficients to fully describe the signal. DWT decomposes the signal into $m+1$ levels, where the level is denoted as $\mathrm{j}$ and the levels are numbered $\mathrm{i}=-1,0,1,2,3 \ldots \mathrm{m}-1$. Each level $\mathrm{i}$ consists of $j=2 \mathrm{i}$ wavelet translated and equally spaced $2 \mathrm{~m} / j$ intervals apart. The $j=2 \mathrm{i}$ wavelets at level $\mathrm{i}$ are dilated such that an individual wavelet spans $\mathrm{n}-1$ of that level interval, where $\mathrm{N}$ is the order of the wavelet being applied. The forward wavelet transform determines $\left(a_{i, j}\right)$ of $\mathrm{j}$ wavelet at each level ' $\mathrm{i}$ ' for the signal $\mathrm{f}(\mathrm{n})$, the DWT is

$a_{i, j}=a_{2^{i}+j} \sum f(n) \cdot \psi_{i, j}(n)$

\section{B. Continuous wavelet transform}

Often it is desired to differentiate between smaller frequency bands than DWT allows. This is possible by using scales that are more closely spaced together than the $2 \mathrm{i}$ relationship and is the basis for the Continuous Wavelet Transform (CWT). For a signal $\mathrm{f}(\mathrm{t})$, CWT determines the coefficients as

$$
\alpha(i, j)=\int_{-\infty}^{+\infty} f(t) \psi(i, j, t) d t
$$

Here $\psi$ is the mother wavelet.

The term wavelet means a small wave. The term wave refers to the condition that this function is oscillatory. The number of coefficients necessary to describe the signal may be larger than the signal strength, as the CWT over samples the signal. The calculation of CWT is usually performed by taking discrete values for scaling parameter and translation parameter.

\section{Application Of Wavelet In Fault Diagnosis}

The use of wavelets for induction machine fault detection is documented in various journals. They have been shown to yield satisfactory results for detecting electrical and Mechanical fault. Wavelet decomposition results in useful data contained in 'details' and 'approximate' parts as shown in the simplified block diagram of Figure1.The 'approximation' signal can be further decomposed in to a new set of 'approximation' and 'details' signals and continue until $n$ decomposition levels. The 'details' signal contains high frequency information whereas the approximate part contains signal data with the low frequency components. Computing this decomposition to $n$ levels results in those higher detail parts being removed, thereby reducing the overall frequency characteristics of the resulting data. This implies that lower Levels of decomposition provide detail data that contains the highest frequency components. For the induction motor signature analysis, the higher frequency wavelet components represent system noise or harmonics due to faults in the dc link of induction motor drive. 


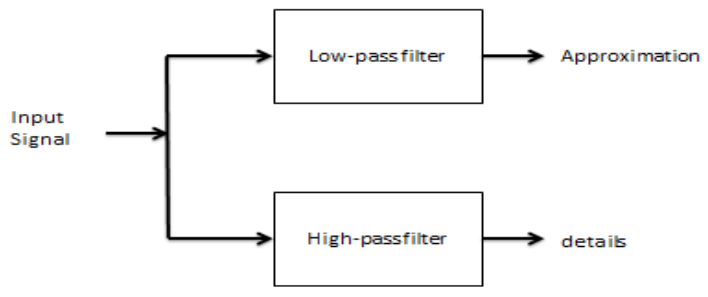

Fig.1 First level decomposition in wavelet

Figure (2) shown below is of reconstructed signal representing decomposition of a signal. Fault patterns are obtained from the information yielded by the $n$-level wavelet decomposition through a variety of strategies, including filter banks and classification algorithms

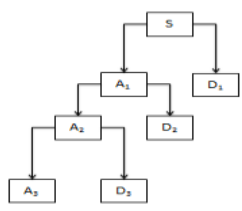

Fig. 2 Reconstructed signal components

\section{Functional Units}

Figure (3) describes the functional units present in induction motor drive. A 5.4 HP induction motor is driven by voltage source inverter. The operation of the system is as follows: A 3 phase supply of $50 \mathrm{~Hz}$ is given to a rectifier which converts the given ac source to dc. The filter circuitry in the dc link is meant to reduce the harmonic content in the ac input and ripples present in the dc output. The filtered dc output is fed to the inverter which converts dc into ac and the output obtained from the VSI is fed to the induction motor. Figure (4) shows the introduction of an open circuit fault and a short circuit fault in the dc link of the induction motor drive. The open circuit fault occurs first for a duration of 0.2 seconds followed by a short circuit fault which occurs across the capacitor in dc link which too lasts for 0.2 seconds. The switching over of the first breaker starts at $1 \mathrm{sec}$ and lasts for 0.2 seconds which forms open circuit fault. The switching over of the second breaker starts at 2 sec and lasts for $0.2 \mathrm{sec}$ and it forms the short circuit fault across the capacitor. The performance of the drive in both the cases is analysed by wavelet theory. The output stator current signal obtained from the induction motor is used for the detection of faults occurring in the system

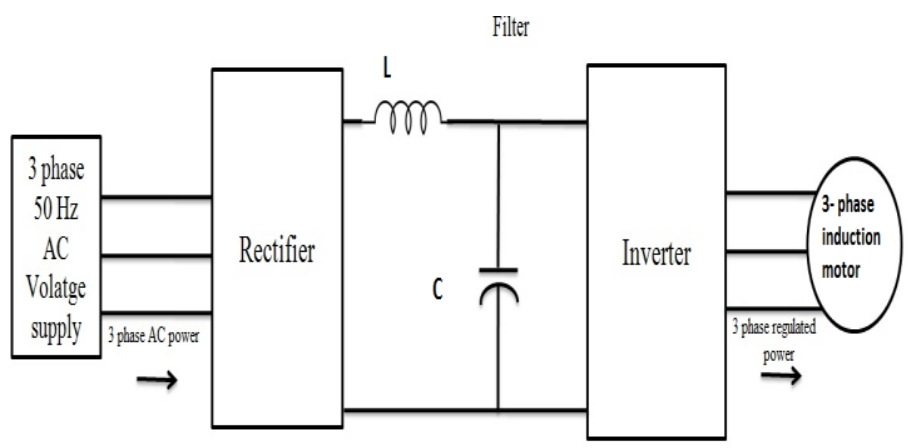

Fig.3 Block diagram of an induction motor drive 


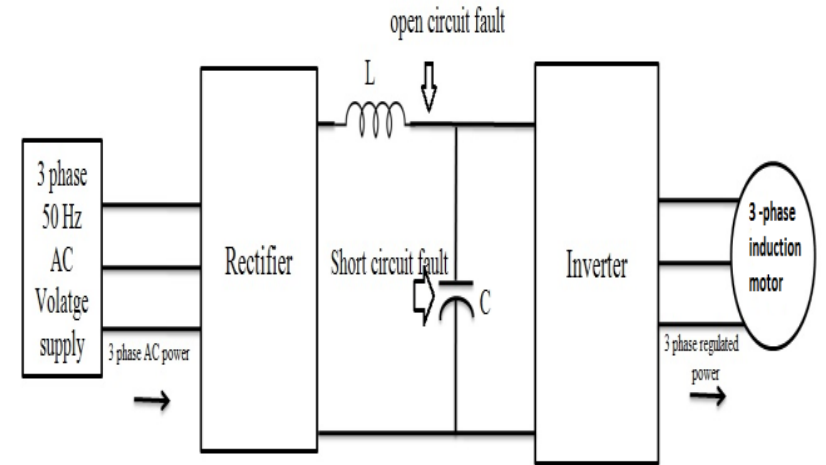

Fig.4 Block diagram of induction motor drive after introduction of faults in de link

\section{Simulation Model}

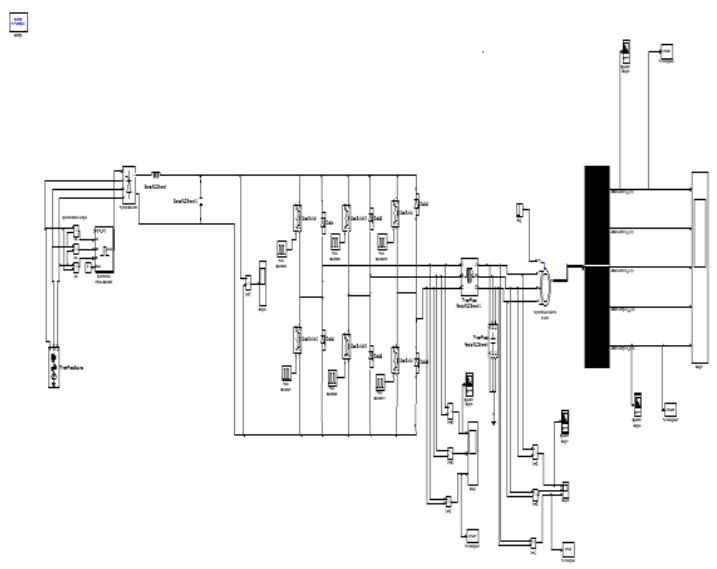

Fig.5 Simulation model of healthy induction motor drive

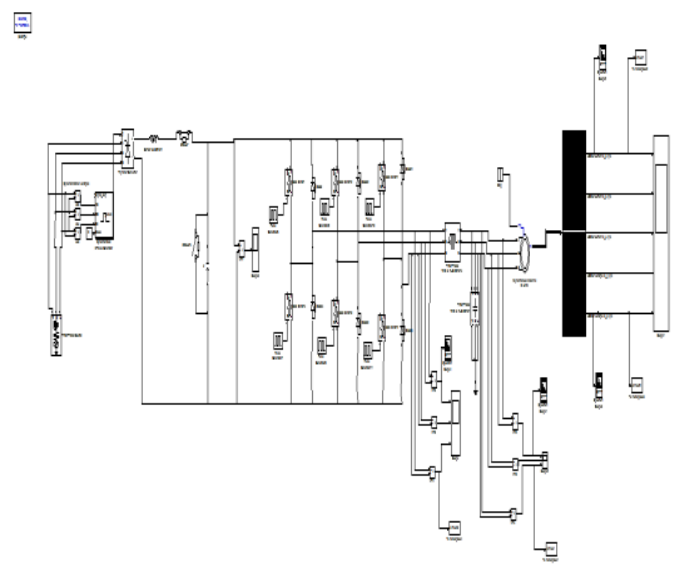

Fig.6 Simulation model of induction motor drive after introduction of faults in dc link

\section{Simulation Results}

Various simulations are done using MATLAB software for the induction motor drive circuit presented in figure (3). The output of the VSI is given to the induction motor. The drive circuit shown in figure (3) is the healthy induction motor drive. Figure (4) shows the induction motor drive circuit here two faults are introduced in the dc link before the inverter circuitry. These two faults are open circuit and short circuit faults. To analyse the change in the operation of the drive after the introduction of faults, the stator current signal of the induction motor is analysed using the wavelet toolbox which is available in Matlab. The stator current signal is analysed \& decomposed using symlet wavelet at the $6^{\text {th }}$ level of decomposition. The figure (7) shows dc output voltage 
waveform of the unhealthy induction motor drive. The figure (8) shows the stator current signal for the healthy induction motor drive. Figure (9) shows stator current signal for the unhealthy induction motor drive. It can be clearly seen that by using wavelet transform the exact location \& frequency of the signal can be detected at the exact instant where the actual discontinuity takes place which also helps in specifying the fault type.

\section{Fault Detection Process}

The wavelet toolbox is able to analyse $\&$ detect the open circuit $\&$ short circuit faults that occur in the dc link of an induction motor drive. The decomposition at level 6 of symlet wavelet is presented to ensure the best detection of both the faults in the dc link.

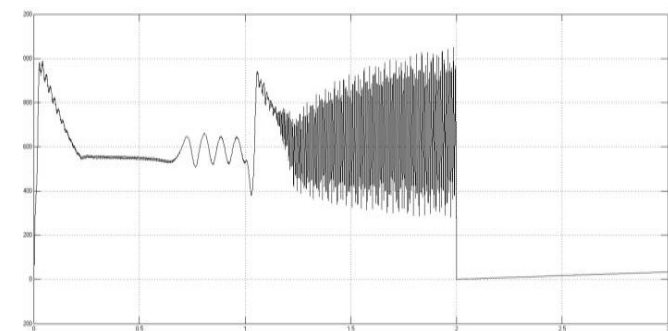

Fig.7 DC output voltage waveform

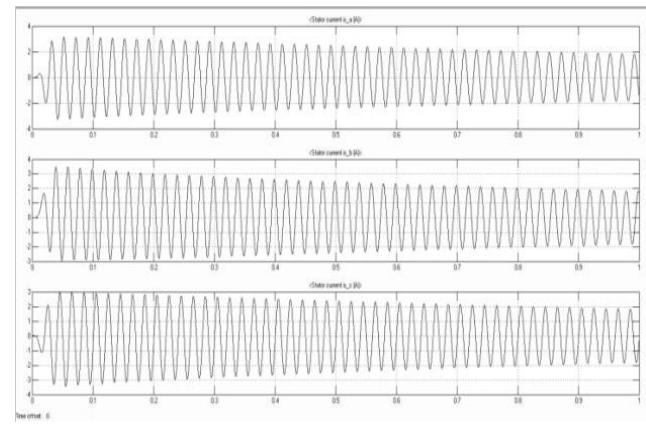

Fig. 8 Stator current signal of healthy induction motor drive

The amplitude of stator current in the case of short circuit fault in the dc link of the induction motor drive is -50 amperes to +50 amperes in faulty state. The approximation \& detail coefficients of the wavelet effectively detect open circuit \& short circuit faults in the dc link of induction motor drive.

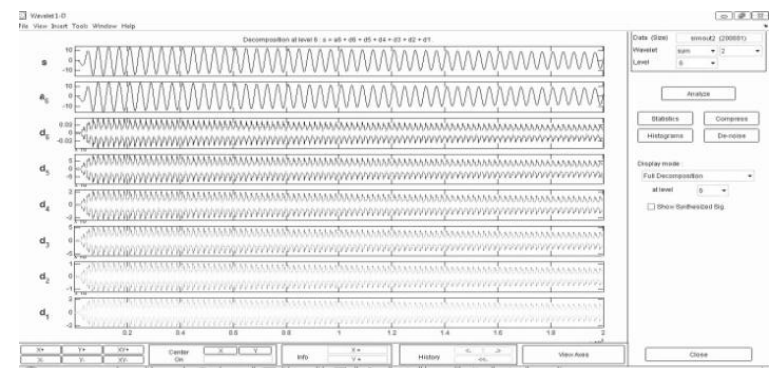

Fig.9 Approximation signal of healthy stator current signal

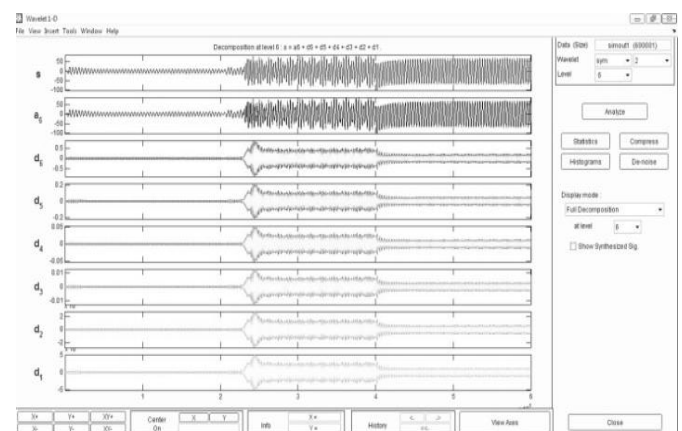

1Fig. 10 Approximation signal of unhealthy stator current signal 


\section{Conclusion}

This paper presents wavelet techniques for the diagnosis of open circuit \& short circuit faults that occur in the dc link of an induction motor drive. The fault detection carried out using wavelet analysis helps to extract fault characteristics.. The result of the symlet wavelet gives advantageous information to decide faulty situation, particularly in case of open circuit \& short circuit faults.

\section{References}

[1]. P. frank; R. Patton and R. Clark., Fault diagnosis in dynamic systems theory and application Prentice hall New Jersey 1989

[2]. P. Frank; R. Patton and R. Clark., Issues in fault diagnosis for dynamic systems Springer Verlag 2000.

[3]. M.E.H Benbouzid, "Bibliography on induction motors faults detection and diagnosis", IEEE Trans. Energy Conversion, vol. 14(4), pp 1065-1074, December, 1999

[4]. Blodt M and Chabert M. and Regnier j Faucher, "Mechanical load fault detection in induction motors by stator current timefrequency analysis", IEEE Trans. Ind. Appl., 2006, 42, (6), pp. 1454-1463.

[5]. W.Yoon and M.J.Devaney, "Reactive power measurement using the wavelet transform", IEEE Trans. Instrum. Meas, vol. 49, no. 2, pp. 579-584, Apr. 2000

[6]. L.Eren and M. J. Devaney, "Bearing damage detection via wavelet packet decomposition of the stator current, ”IEEE Trans. Instrum. Meas., vol. 53, no. 2, pp. 431-436, Apr. 2004

[7]. Nandi S.; Toliyat H.A. "Condition monitoring and fault diagnosis of electrical machines - a review", IEEE Trans. Energy Convers. 20, (4), pp. 719-729 2005

[8]. C. Wang and R. Gao., "Wavelet transform with spectral post-processing for enhanced feature extraction”,IEEE Trans. Instrum. Meas., vol. 52, no. 4, pp. 1296-1301, Aug. 2003

[9]. B. Akin.;U. Orguner.;H.A Toliyat.;M. Rayner, "Low order PWM inverter harmonics contributions to the inverter-fed induction machine fault diagnosis," IEEE Trans. Ind. Electron., vol. 55(2), pp 610-619, Frebruary, 2008 